\title{
Severe Acute Rhabdomyolysis Induced by Multi-Agent Chemotherapy for Alveolar Rhabdomyosarcoma in a 15-Year-Old Female: A Case Report
}

\author{
Hiroshi Matsuzaki Yuhki Koga Aiko Suminoe Utako Oba \\ Tomohito Takimoto Toshiro Hara \\ Department of Pediatrics, Graduate School of Medical Sciences, Kyushu University, \\ Fukuoka, Japan
}

\section{Key Words}

Rhabdomyolysis $\cdot$ Chemotherapy $\cdot$ Rhabdomyosarcoma $\cdot$ Child $\cdot$ Acute renal failure

\section{Abstract}

This is the first paper to report the association of cancer chemotherapy with rhabdomyolysis in children. A previously healthy, 15-year-old Japanese female was diagnosed as having alveolar rhabdomyosarcoma. She received the first cycle of multi-agent chemotherapy without any adverse effects. However, she developed severe acute rhabdomyolysis shortly after the second cycle of multi-agent chemotherapy, which consisted of etoposide, ifosfamide, actinomycin-D and vincristine. Her condition deteriorated rapidly and she was treated with mechanical ventilation and fluid replacement. After further evaluation, anticancer drugs were thought to be responsible for the rhabdomyolysis.

\section{Introduction}

Rhabdomyolysis is a syndrome caused by leakage of large quantities of muscle cell contents including electrolytes, myoglobin and other sarcoplasmic proteins into the blood, 
following the necrosis of skeletal muscle [1]. This manifests as limb weakness, myalgia and swelling, usually accompanied by gross pigmenturia without hematuria. Acute renal failure (ARF) is the most important complication of severe rhabdomyolysis, which results in worse prognosis of the patient $[2,3]$. In previous studies, the rates of ARF secondary to rhabdomyolysis range from 42 to $50 \%$ in children $[1,4]$. There are various causes of acute rhabdomyolysis in childhood such as trauma, infection, connective tissue disease, ischemia-induced muscle necrosis, inflammation in muscle and exposure to drugs and/or toxins. The association of cancer chemotherapy with rhabdomyolysis has not been reported in children.

Here, we describe a 15-year-old female with alveolar rhabdomyosarcoma who developed severe acute rhabdomyolysis shortly after chemotherapy.

\section{Case Report}

A previously healthy, 15-year-old Japanese female presented with right nasal congestion and a right submandibular mass. On admission, physical examination revealed only a submandibular mass (approximately $2 \mathrm{~cm}$ in diameter), which was solid, painless and immovable; no other remarkable abnormalities were found. Laboratory findings were all within normal limits: blood urea nitrogen (BUN) $10 \mathrm{mg} / \mathrm{dl}$, creatinine $0.58 \mathrm{mg} / \mathrm{dl}$, aspartate aminotransferase (AST) $34 \mathrm{U} / \mathrm{l}$, alanine aminotransferase (ALT) $16 \mathrm{U} / \mathrm{l}$, lactate dehydrogenase (LDH) $533 \mathrm{U} / \mathrm{l}, \mathrm{C}$-reactive protein (CRP) $0.02 \mathrm{mg} / \mathrm{dl}$, and creatine kinase (CK) $92 \mathrm{U} / \mathrm{l}$. Magnetic resonance imaging showed a mass in the right maxillary sinus with hyperintensity on T1- and T2-weighted images, which were enhanced by gadolinium. FDG-positronemission tomography and bone and Ga scintigraphy revealed multiple metastases in the right submandibular and bilateral cervical lymph nodes, and bones (thoracic and lumbar vertebrae, right humerus and right sacroiliac region). Bone marrow metastasis was not detected. Histological examination showed monotonous proliferation of small round tumor cells, which were positive for desmin and HHF35. PAX3-FKHR gene alteration was positive in real-time polymerase chain reaction. These findings indicated a diagnosis of alveolar rhabdomyosarcoma (stage 4 of the IRS-V TNM staging classification, group IV of the IRS clinical grouping classification).

She received etoposide, cyclophosphamide, pirarubicin, vincristine and cisplatin as her first cycle of chemotherapy without any adverse effects. Her CK level was within normal range during this cycle of therapy. The second cycle of chemotherapy consisted of etoposide $\left(100 \mathrm{mg} / \mathrm{m}^{2}\right.$ intravenously for 5 days), ifosfamide $\left(1,800 \mathrm{mg} / \mathrm{m}^{2}\right.$ intravenously for 5 days), actinomycin-D (0.015 mg/kg intravenously for 5 days) and vincristine $\left(1.5 \mathrm{mg} / \mathrm{m}^{2}\right.$ intravenously on days 1 and 8). Twenty-four hours after the end of chemotherapy, she developed a high fever, generalized myalgia and pigmenturia. After 4 days, her condition deteriorated rapidly. She suffered from disturbance of consciousness, respiratory failure, ARF, acute liver dysfunction and disseminated intravascular coagulation. She was treated with mechanical ventilation and fluid replacement. The laboratory examination showed; CK 114,268 U/l (CKMB 0.16\%), BUN $41 \mathrm{mg} / \mathrm{dl}$, creatinine $3.01 \mathrm{mg} / \mathrm{dl}$, total bilirubin $7.8 \mathrm{mg} / \mathrm{dl}$, AST 1,187 U/l, ALT $204 \mathrm{U} / \mathrm{l}$, LDH 4,330 U/l, CRP $22.13 \mathrm{mg} / \mathrm{dl}$, fibrin degradation products $10.6 \mu \mathrm{g} / \mathrm{ml}$ and D-dimer $3.1 \mu \mathrm{g} / \mathrm{ml}$. These findings were consistent with rhabdomyolysis. The estimated glomerular filtration rate (eGFR) using serum creatinine was $27.1 \mathrm{ml} / \mathrm{min} / 1.73 \mathrm{~m}^{2}$. Even after extubation, she remained in an oliguric state and was bedridden due to severe muscle atrophy. She was treated with continuous hemodiafiltration or continuous ambulatory peritoneal dialysis (CAPD). 
In order to search for the cause of rhabdomyolysis, we performed magnetic resonance imaging, evoked electromyography, nerve conduction study, a muscle biopsy from the right musculus quadriceps femoris, bacterial cultures and additional laboratory tests. Evoked electromyography and nerve conduction study showed no indication of neuropathy. The biopsy section showed marked size variation of muscle fibers with occasional central nuclei. There were basophilic muscle fibers, suggestive of regeneration of damaged fibers in the frozen sections. Small angular fibers and a nuclear clamp were also seen. A mild endomysial mononuclear cell infiltrate was noted. Necrotic muscle fibers were not evident. These histological features of the muscle biopsy were compatible with the regenerative process of rhabdomyolysis. A high CRP level suggested severe infection, although all bacterial cultures were negative. The final diagnosis was nontraumatic rhabdomyolysis, and anticancer drugs were thought to be responsible for rhabdomyolysis because other possible causes were unlikely. However, the particular causative drug was unclear.

After discontinuing CAPD, the patient's conditions improved slowly, and low-dose chemotherapy (vincristine, actinomycin D and cyclophosphamide) and local radiation therapy were started 5 months after the episode of rhabdomyolysis. Forty-eight months after the initial presentation, she maintained complete remission. Her renal function has improved to the latest eGFR of $96.5 \mathrm{ml} / \mathrm{min} / 1.73 \mathrm{~m}^{2}$, although it still takes some effort for her to walk and she continues to receive rehabilitation.

\section{Discussion}

The classic symptomatic triad of rhabdomyolysis includes myalgia, weakness and dark urine, although these findings may not always be consistent. The definitive diagnosis of rhabdomyolysis requires an elevation of $\mathrm{CK}$ levels to $>5$ times the normal range in the absence of significant elevations of brain or cardiac CK fractions [3]. Gabow et al. [5] showed that the most common causes of rhabdomyolysis in adults included alcohol abuse (67\%), recent soft tissue compression (39\%) and seizure activity (24\%). Ward [6] also showed that trauma (38\%), ischemia (14\%) and polymyositis (24\%) were major causes. However, Mannix et al. [4] showed that the risk factors for rhabdomyolysis in children are different than those in adults, including viral myositis (38.2\%), trauma (25.7\%), connective tissue disease (5.2\%), drug overdose (4.2\%), exercise (4.2\%), metabolic disorder (3.7\%), seizure (3.7\%), sepsis (3.1\%), drug reaction (2.1\%) and hypoxia (2.1\%). Most notably, viral myositis was the most common cause of rhabdomyolysis in patients $<9$ years of age, whereas trauma and drugs were the major causes in children $>9$ years of age and adults.

As mentioned above, drug-induced rhabdomyolysis is very rare in children. In particular, rhabdomyolysis associated with anticancer drugs has not been reported. This may be related to the fact that striated musculature, in contrast to other tissues such as liver and kidneys, exhibits little affinity for most drugs [7]. In adults, there are several reports of rhabdomyolysis associated with anticancer drugs. The anticancer drugs and their combinations which cause rhabdomyolysis include ET-743 [8], paclitaxel and gemcitabine [9], cytarabine [10], high-dose ifosfamide, carboplatin and etoposide [11], stem cell transplantation conditioning using thiotepa and melphalan [12], doxorubicin, cytarabine, thioguanine and vincristine [13], mitoxantrone and cyclophosphamide [14], pemetrexed [15], high-dose cyclophosphamide [16], vincristine, cytarabine and adriablastin [17], and 5-azacytidine [18] (table 1).

The mechanism of anticancer drug-induced rhabdomyolysis is unclear. Cytarabine may interfere with cellular energy metabolism in mitochondria [13]. Alkylating agents (e.g. 
cyclophosphamide and ifosfamide), anthracyclines (doxorubicin, etc.) and anthracenediones (mitoxantrone, etc.) induce cardiac muscle damage, although skeletal muscle damage is uncommon $[14,16]$. Anthracyclines and anthracenediones induce the generation of oxygen free radicals leading to cardiac muscle injury, although it is unclear why a similar phenomenon does not occur in skeletal muscle [14]. Cyclophosphamide-induced muscle damage is enhanced by a glutathione synthesis inhibitor in mice [16]. Taxanes may provoke myositis, sometimes in a severe form, and incidence reaches up to $2 \%$ when they are used in combination with gemcitabine [9]. In most cases, rhabdomyolysis usually occurs within 1-3 days of chemotherapy [14].

In our patient, common causes for rhabdomyolysis including trauma, infection, connective tissue disease, drug overdose, exercise, metabolic disorder, seizure and sepsis were either excluded or not evident. Therefore, anticancer drugs were thought to be responsible for the development of rhabdomyolysis, although the particular causative agent is unclear. Recently, common variants in SLCO1B1 were found to be strongly associated with an increased risk of statin-induced myopathy by a genome-wide study on the mechanism of drug-induced myopathy [19]. To date, no studies on the genetic association between rhabdomyolysis and anticancer drugs have been reported.

Rhabdomyolysis is a rare complication in childhood, especially in patients receiving cancer chemotherapy. This disease is usually thought to be benign in children, with only a small risk of ARF [4]. However, rhabdomyolysis-induced ARF is a very important complication in children as well. Several mechanisms by which rhabdomyolysis leads to acute kidney injury (AKI) are proposed, including direct renal tubular toxicity of myoglobin, intrarenal vasoconstriction and tubular obstruction, as well as secondary injury associated with volume depletion and renal ischemia. In addition, several adult studies have evaluated predictive factors of rhabdomyolysis-induced AKI. These studies suggest that the presence of dehydration, metabolic acidosis with aciduria, oliguria, massive muscle damage, higher illness severity and systemic inflammatory response syndrome are predisposing factors for the development of AKI with rhabdomyolysis [20]. In our patient, some of the proposed mechanisms are far from impossible, but none of the predisposing factors were evident. Rhabdomyolysis-induced AKI/ARF is potentially life-threatening and leads to the delay of chemotherapy. Although the dose range of most anticancer drugs can be limited by renal function, many anticancer drugs can induce renal injury and rhabdomyolysis, which may lead to AKI/ARF. This is a paradox which exists during chemotherapy. Therefore, it is important that we be aware of the possibility of drug-induced rhabdomyolysis and make preemptive medical decisions to prevent rhabdomyolysis in high-risk patients during cancer chemotherapy.

\section{References}

1 Watanabe T: Rhabdomyolysis and acute renal failure in children. Pediatr Nephrol 2001;16:1072-1075.

2 Bosch X, Poch E, Grau JM: Rhabdomyolysis and acute kidney injury. N Engl J Med 2009;361:62-72.

-3 Goldsmith BM, Hicks JM: Rhabdomyolysis: two pediatric case reports. Clin Chem 1985;32:314-317.

4 Mannix R, Tan ML, Wright R, Baskin M: Acute pediatric rhabdomyolysis: causes and rates of renal failure. Pediatrics 2006;118:2119-2125.

5 Gabow PA, Kaehny WD, Kelleher SP: The spectrum of rhabdomyolysis. Medicine (Baltimore) 1982;61:141152.

6 Ward MM: Factors predictive of acute renal failure in rhabdomyolysis. Arch Intern Med 1988;148:15531557.

7 Köppel C: Clinical features, pathogenesis and management of drug-induced rhabdomyolysis. Med Toxicol Adverse Drug Exp 1988;4:108-126. 


\section{Case Reports in Oncology}

\begin{tabular}{l|l}
\hline Case Rep Oncol 2013;6:397-402 \\
\hline DOI: $10.1159 / 000354271$ & $\begin{array}{l}\text { C 2013 S. Karger AG, Basel } \\
\text { www.karger.com/cro }\end{array}$ \\
\hline
\end{tabular}

Matsuzaki et al.: Severe Acute Rhabdomyolysis Induced by Multi-Agent Chemotherapy for Alveolar Rhabdomyosarcoma in a 15-Year-Old Female: A Case Report

8 Skorupa A, Beldner M, Kraft A, Montero AJ: Fatal rhabdomyolysis as a complication of ET-743 (Yondelis) chemotherapy for sarcoma. Cancer Biol Ther 2007;6:1015-1017.

-9 Vicente E, Zafra M, Garcia-Martinez E, de la Peña FA: Acute rhabdomyolysis as a complication of paclitaxelgemcitabine chemotherapy for ovarian cancer. Eur J Obstet Gynecol Reprod Biol 2009;145:226.

10 Truica Cl, Frankel SR: Acute rhabdomyolysis as a complication of cytarabine chemotherapy for acute myeloid leukemia: case report and review of literature. Am J Hematol 2002;70:320-323.

11 Hoshi S, Itoh A, Kato S, Suzuki K, Kawamura S, Orikasa S: Severe rhabdomyolysis as a complication of highdose chemotherapy in a patient with advanced testicular cancer. Int J Urol 1999;6:56-58.

12 Pugliese P, Danova M, Brugnatelli S, Piccolo G, Riccardi A, Ascari E: Acute rhabdomyolysis after high dose chemotherapy and circulating progenitor cell autografting for breast cancer. Haematologica 2000;85:672.

13 Papakonstantinou C, Papanastasiou K, Kotsopoulou M, Mouratidou M, Sotiropoulos D, Kyrtsoni MC, Pouli A, Stamatelou M, Maniatis A: Chemotherapy-related acute rhabdomyolysis. J Natl Cancer Inst 1992;84:536537.

14 Levy RJ, Sparano JA, Khan G: Rhabdomyolysis: an unusual complication of cytotoxic chemotherapy. Med Oncol 1995;12:219-222.

-15 Ceribelli A, Cecere FL, Milella M, Facciolo F, Gelibter A, Cognetti F: Severe rhabdomyolysis associated with pemetrexed-based chemotherapy. Lancet Oncol 2006;7:353.

16 Shima E, Hino M, Yamane T, Aoyama Y, Nakamae H, Yamamura R, Makita K, Sugano Y, Yasuda S, Takubo T, Ohta K, Tatsumi N: Acute rhabdomyolysis following administration of high-dose cyclophosphamide: case report. Ann Hematol 2002;81:55-56.

17 Luliri P, Bobbio-Pallavinci E, Gorini M: Acute rhabdomyolysis during treatment with epsilon aminocaproic acid. Description of two cases. Haematologica 1983;68:664-669.

18 Koeffler HP, Haskell CM: Rhabdomyolysis as a complication of 5-azacytidine. Cancer Treat Rep 1978;62:573-574

19 SEARCH Collaborative Group, Link E, Parish S, Armitage J, Bowman L, Heath S, Matsuda F, Gut I, Lathrop M, Collins R: SLC01B1 variants and statin-induced myopathy - a genomewide study. N Engl J Med 2008;359:789-799.

20 Al-Ismaili Z, Piccioni M, Zappitelli M: Rhabdomyolysis: pathogenesis of renal injury and management. Pediatr Nephrol 2011;26:1781-1788. 
Matsuzaki et al.: Severe Acute Rhabdomyolysis Induced by Multi-Agent Chemotherapy for Alveolar Rhabdomyosarcoma in a 15-Year-Old Female: A Case Report

Table 1. Rhabdomyolysis associated with chemotherapy

\begin{tabular}{|c|c|c|c|c|c|}
\hline Drug & $\begin{array}{l}\text { Age, } \\
\text { years }\end{array}$ & Sex & Disease & Interval & Ref. \\
\hline ET-748 & 44 & Male & Liposarcoma & 9 days & 4 \\
\hline $\begin{array}{l}\text { Paclitaxel } \\
\text { Gemcitabine }\end{array}$ & 64 & Male & Ovarian papillary serous adenocarcinoma & 19 days & 5 \\
\hline Cytarabine & 47 & Male & Acute myeloid leukemia & 7 days & 6 \\
\hline $\begin{array}{l}\text { Ifosfamide } \\
\text { Carboplatin } \\
\text { Etoposide }\end{array}$ & 38 & Male & Testicular cancer & 3 days & 7 \\
\hline $\begin{array}{l}\text { Thiotepa } \\
\text { Melphalan }\end{array}$ & 47 & Female & Breast cancer & 7 days & 8 \\
\hline $\begin{array}{l}\text { Doxorubicin } \\
\text { Cytarabine } \\
\text { Thioguanine } \\
\text { Vincristine } \\
\text { Methylprednisolone }\end{array}$ & 55 & Female & Acute myelomonocytic leukemia & 1 day & 9 \\
\hline $\begin{array}{l}\text { Mitoxantrone } \\
\text { Cyclophosphamide }\end{array}$ & 46 & Female & Breast cancer & 6 days & 10 \\
\hline Pemetrexed & 68 & Male & Mesothelioma & 2 days & 11 \\
\hline Cyclophosphamide & 47 & Female & Adult T-cell leukemia & $19 \mathrm{~h}$ & 12 \\
\hline $\begin{array}{l}\text { Epsilon-aminocaproic } \\
\text { acid }\end{array}$ & & & & & 13 \\
\hline 5-azacytidine & 26 & Female & Breast cancer & 6 days & 14 \\
\hline
\end{tabular}

